tomed, howerer, to pay much attention to the history of the case, but to judge each on its merits, as I see it, though it may be taken as a rule to which there are few excep. tions, that in severe cases the severity of the disease directly depends upon its duration. Severity of attack is shown by extent of exudation and symptoms of toxaemia.

The dose I am accustomed to give is from 2,000 units for a mild case up to 20,000 for a severe case, repeating half this amount the next day if there is any increase in the severity of the disease. In most instances this will be sufficient, but in very severe cases, especially of the haemorrhagic form, large doses should be injected. In one sense you cannot give too much serum ; you cannot poison a person by an overdose, as you can with such drugs as morphine and strychnine. Nor is it necessary to take into consideration the age of the patient, unless it be to reverse the ordinary principles of dosage by giving larger amounts of antitoxin to children than to adults.

I have always administered serum by subcutaneous injection. No doubt the most efficacious method is by intravenous injection; but it presents considerable practical difficulties, of which the chief is that it is by no means easy to pick out a vein in a small child who is the subject of diphtherial toxaemia. Of administration by the mouth or rectum, advocated by some writers, I have no experience. I am certain that by hypodermic injection the serum will be retained and absorbed, bat I am not so sure that it will be, in any given case, if administered by the channels mentioned.

Lastly, I will just touch on one question that may be asked, namely, can the unpleasant reactions I have been talking about be in any way prevented? So far as I know, they cannot; there is conflict of evidence on the subject. But neither heating the serum nor the use of any drug appears to be entirely efficacions, though there is clinical evidence to show that large doses of calcium lactate will mitigate the rash of serum sickness. Anaphylaxis can be produced in animals by foeding them on certain proteins, so that the rectal or oral administration cannot be expected to be free from risk of the unpleasant sequels. Bat this I will sey, that it would seem as if there were some methods in the manufacture of the serum that were better than others so far as the avoidance of unpleasent effects is concerned ; for certainly during the past few years the antitoxic serum supplied to the Asylums Board's hospitals has been less noxious than it was at one time. I have not, however, been able to ascertain the cause of the undoubted improvement that has been effected.

REFERENCES.

1 Die Serumkrankheit, 1905. 2 BRITISH MEdICAL JouRnaL, February 29th, 1908. ${ }^{8}$ Ibid.. January 18th, 1908 . \& Reported in Appendix III to the Report of the Clinical Society on Antitoxin, 1898. B Glasgow Medica Journal, April, 1908. The reaction was local and slight. 6 Berl. klin. 8 Therapeutic Gazette, March 15th Amer. Med. As80e., March 15th, 1908. 29th, 1908. 10 Journ. Exper. Med., September lst, 1910. 11 Bulletin Johns Hopkins Hospital, September, 1910.

A PRELIMINARY report by Captain Stevenson, I.M.S (The Scientiflc Memoirs by Officers of the Medical and Sanitary Departments of the Government of India; new series, No. 38, 1910), on the killing of rats and rat fleas by hydrocyanic gas has been issued recently. Fumigation with this gas has been largely used by the agricultural departments in the United States of America and in South Atrica, chiefly for the destruction of scale insects infesting plants. To produce the gas, an enamel ware vessel or a kerosene tin is placed in the middle of the room to be fumigated. Measured quantities of water and sulphuric acid are then placed in this, and when all is ready the potassium cyanide is dropped in, the operator bolting from the room as fast as he can get out. The man who measures out the potassium cyanide must use rubber gloves, otherwise he will almost certainly be poisoned, and the individual who drops it into the water and acid should do so at arm's length, retiring, as has already been caid, as fast as he can. So deadly is the gas, that a whiff of fairly strong vapour kills at once. Further, as it is somewhat inflam. mable, no naked lights must be left about the buildings to be fumigated. Apparently the gas is harmless to the plague bacilli themselves, but is very toxic to fleas and their hosts the rats. The risk attending the employment of such a deadly gas as this would seem to be too great, at least for general use.

\section{THE ARGYLL ROBERTSON SIGN IN CEREBRAL AND SPINAL SYPHILIS.}

J. MICHELL CLARKE, M.A., M.D.Cantab, F.R.C.P,, PROFESSOR OF MEDICINE, UNIVERSITY OF BRISTOL; PHYSICIAN TO BRISTOL GENERAI HOSPITAL.

The frequency and importance of the Argyll Robertson sign in tabes dorsalis and general paralysis is a matter of general knowledge, bat there still seems some doubt as to its incidence in cerebro-spinal syphilis. In $\mathrm{Dr}$. Risien Rassell's ${ }^{1}$ very interesting opening address to the discussion on ophthalmoplegia at the Annual Meoting of the British Medical Association last year, he asked the pertinent questions as to how far persons who have had syphilis and have the Argyll Robertson sign have escaped tabes or general paralysis, and whether this sign is compatible with a syphilitic lesion which does not mean a tendency to progressive degeneration. It was disappointing that in the discussion which followed no decisive answers were given to these questions, desirable as it is that a point of such diagnostic importance should be settled.

Dr. Mott, 2 in a discussion on syphilis in the Neurological Section of the Royal Society of Medicine, said on this point: "There is one sign usually present which for all practical purposes is only met with in parasyphilisnamely, the Argyll Robertson pupil. No coarse random lesion will explain the constancy of this phenomenon; moreover, this condition, although a sign of syphilitic infection, does not occur in true syphilitic brain disease."

The Argyll Robertson phenomenon is to be regarded as an example of the selective action of a poison upon the central nervous system, inasmuch as a special group of neurones having a definite and restricted function is picked out and put out of action. It may be compared in this respect with the action of the diphtheritic poison in paralysing accommodation. The parasyphilitic affections offer other examples of similar selective action of the virus-for example, the disease of certain fibres of the pos. terior roots in tabes. In cerebro-spinal syphilis, however, such results are conspicuously absent, the paralyses there met with are produced as the conseguence of interference with the nutrition of the neurones by occlusion of vessels from endosrteritis or thrombosis, by their compression by exudation, or by destraction through gummatous tumours. $A$ priori, therefore, it would be unlikely that a sign like the Argyll Robertson papil should occur in cerebro-spinal syphilis.

In 1903, in a paper ${ }^{3}$ on this subject based on the analysis of 37 cases of syphilis of the brain and cord, I concluded that previous syphilis is not sufficient without some further change to lead to the presence of this sign: that its presence is evidence of a degenerative process at work within the nervous system, and this process is to be regarded as a "parasyphilitic" one; but that it is reasonable to suppose that the degenerative process, of which the Argyll Robertson sign is one of the symptoms, may remain stationary at an early stage, or for a long period without further development. An exampie of this arrest is seen in some cases of tabes dorsalis. With some exceptions to be mentioned later, this view, if correct, would practically confine the sign to parasyphilitio infections.

In a recent analysis of 48 cases of syphilis of the brain and 21 of the cord, 69 in all, I obtained the following resalts with regard to the Argyll Robertson pupil. In every case there was a clear history of syphilis, or some undoubted syphilitic lesion, and, in addition, in the later cases Fleming's test was positive. Ont of the 69 cases, the Argyll Robertson pupil was present in 2 of corebral syphilis. One of these was a man of 32 , apparently with syphilitic meningitis of the convexity; he had headaches, was apathetic forgetful depressed, with indistinct or slightly slurring speech and exaggerated knee-jerks. He showed no improvement nnder treatment, and his symptoms suggested the possibility of an early stage of general paralysis. The other patient was a woman, aged 45, who suffered from right facial paralysis twelve years ago, five years ago was under my care for corebral syphilis, with right hemiplegia and 
Argyll Robertson pupil, and has developed on the hemiplegia a typical tabes dorsalis. In another patient there was paralysis of the right third nerve, with complete iridoplegia, the left pupil did not react to light on admission, but recovered fully under treatment. In six pationts the pupil reaction to light was sluggish, but not absent, and in three of them became much more brisk under treatment. One of these suffered from epileptiform fits, both general and localized, with headache and vomiting: two from hemiplegia, with headache and vomiting; one from meningitis of the convexity, with hemiparesis, cranial nodes, and double optic neuritis; the fifth was a patient with hereditary syphilis, with cerebral symptoms and optic neuritis; and the sixth was a patient suffering from syphilitic cerebro-spinal meningitis, with hemiplegia and intense optic nenritis. So that optic neuritis, in vary ing intensity, was present in three of the six cases with a sluggish reaction; optic neuritis does not, of course, abolish the light reaction. Of the 21 spinal cases, in one the light reaction was sluggish, and in two the Argyll Robertson sign was present. Of the latter, one was a rather remarkable case. The patient was a man, aged 32 , who had had left hemiplegia two years betore, trom which recovery was not complete. When admitted, he was dull, apathetic, and had some mild delusions as to place and as to his circumstances. The cord affection corresponded to Erb's syphilitic spinal paralysis, and was of very gradual onset, with the usual affection of the bladder, great exaggeration of deep reflexes, and double extensor plantar reflex. The pupils were very small. He made, under mercurial treatment, an nnexpectedly good recovery, both as to his mental and physical state, and was able to retarn to work. The Argyll Robertson sign persisted. In the other case the cord affection was of the same kind, with no affection of sensation, no ataxy, and no symptoms except slight incontinence beyond those clinically referable to degeneration of the pyramidal tracts; the disease was also of very gradual onset.

It is an interesting point that the only cases presenting the Argyll Robertson sign amongst my cases of syphilitic disease of the cord are cases belonging to the clinical group of syphilitic spastic paraplegia, first described by Erb. Wilfred Harris ${ }^{4}$ states that in Erb's syphilitic spinal paralysis "sluggishness of pupils, or even complete reflex iridoplegia may, however, be met with." Although presenting the same clinical features, it is probable that these cases do not all own the same pathology. If some of them are due to a primary degeneration of the pyramidal tracts as a result of syphilis, then they would represent a degeneration of the efferent neurones analogous to that of the afferent fibres in tabes, and come under the parasyphilitic affections. Harris quotes Wimmer as stating that in only 4 cases examined pathologically was there a pure degeneration of the pyramidal tracts, but it may be added that the records of autopsies are few. From the recorded cases Nonne ${ }^{5}$ gives the following four conditions as underlying this form of paraplegia: (1) Chronic myelitis in patches, with ascending and descending degenerations; (2) these changes combined with a primary degeneration of the pyramidal tracts; (3) degeneration of the pyramidal tracts only; and (4) a combined primary postero-lateral tract degeneration.

I suggest that it is with the group of pure degeneration of the pyramidal tracts that the Argyll Robertson sign will be found associated, and that the condition is a parasyphilitic one.

In connexion with syphilitic spinal disease, it may be added that Dr. W. Harris ${ }^{6}$ found "complete or partial Argyll Robertson pupil "in three cases of chronic syphilitic poliomyelitis. These cases were a very late sequel of syphilis, in one of the three appearing twenty-five years after infection.

In analysing the above cases, I have not taken into account a unilateral loss of the light reaction, because it is then more difficult to exclude local causes; in four or five of the cases unilateral loss occurred in association with paralysis (complete or incomplete) of the third nerve on the same side. Moreover, if the Argyll Robertson sign is rightly considered as the selective action of a poison, both pupils should be affected. So far as my cases go, therefore, they entirely support the contention that this sign is not a symptom found in pure cerebro-spinal syphilis, and that its presence is an index of a degenerative process at work in the nervous system.

The contrast with tabes and general paralysis, in which the sign is present in about 70 per cent. or over of the cases, is too striking to call for further remark.

In testing the cases no further precaution was taken than always to place the patient opposite a good light (daylight), cover both eyes, and see that they did not accommodate.

I have referred throughout to the fally developed Argyll Robertson sign only, because merely sluggish pupils, although in certain connexions suggestive, have not the same clinical significance. Further, it is now certain that in recent cases and under efficient mercurial treatment, an absent light reflex may sometimes be regained.

There are one or two other conditions under which the Argyll Robertson sign may be present, but the associated symptoms should prevent any error in diagnosis. Thus, Dr. Farquhar Buzzard, ${ }^{7}$ in the discussion on ophthalmoplegia, mentioned cases in which the sign was present in cysts of the third ventricle, and in tumours of the optic thalamus extending to the corpora quadrigemina. Other observers have also noted it in lesions of the corpora quadrigemina, but it does not invariably accompany them. It is also present in optic atrophy, but not in optic neuritis.

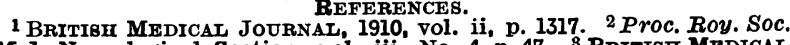
Med., Neurological section, vol. iii, No. 4, p. 47. 3 BRITISH MEDICAL JoURNAL, 1903. vol. ii, p. 1634. 4 Allbutt and Rolleston's System of

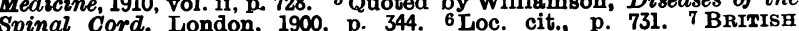
Spinal Cord, London, 1900, p. 34 . 1319.

\section{TUBERCULIN DISPENSARIES.} BY

A. M. N. PRINGLE, M.B., C.M., D.P.H., MEDICAL OFFICER OF HEALTH, CODNTY BOROUGH OF IPSWICH.

IN order to determine the attitade of those members of the medical profession who are specially competent to express an opinion upon the subject, inquiries have been made by the medical superintendents of most of the principal sanatoriums of England and Wales as to the value attached by them to the ase of tuberculin.

The replies received show clearly that taberculin is a remedy viewed with favour by the large majority of the medical superintendents of the sanatoriums of the country. The view of Dr. Wilkinson and Dr. Fraser, M.O.H. Portsmouth, that taberculin is a usefal therapentic agent in the treatment of pulmonary consumption, is thus endorsed.

When we consider the types of cases suitable for the administration of tuberculin, we are confronted with considerable diversity of opinion. Broadly speaking, how. ever, we may conclude that the majority of competent observers are agreed that the most suitable cases are the early, not markedly febrile, group, and that the remedy is contraindicated in mixed infections and in acute febrile cases. In other words, expert opinion inclines to the view that the cases suitable for the administration of taberculin must be carefally selected.

In the next place the question was asked whether tuberculin was a remedy which might be safely and properly administered at a tuberculin dispensary where the patients attend once or twice a week for the purpose of receiving injections. To this question the replies received were practically unanimously in the negative, the opinion being again and again expressed that tuberculin as a therapentic agent ought only to be administered with caution and under conditions of the most constant and careful control.

The fourth question asked was whether the establish. ment of tuberculin dispensaries could replace sanatoriums. To this question the answers were emphatically in the negative.

Finally it was asked whether the best conditions for the administration of tuberculin were not such as obtain in sanatoriums where the patient is under constant skilled supervision and control. In every case the answer was in the affirmative, the opinion being frequently stated that these conditions were precisely those which justified the use of tuberculin for therapentic purposes. 